Pacific Journal of Mathematics

SOME INTEGRAL FORMULAS FOR CLOSED
HYPERSURFACES IN RIEMANNIAN SPACE 


\title{
SOME INTEGRAL FORMULAS FOR CLOSED HYPERSURFACES IN RIEMANNIAN SPACE
}

\author{
Chuan-Chin Hsiung
}

Introduction. Let $V^{n}$ be a hypersurface twice differentiably imbedded in a Riemannian space $R^{n+1}$ of $n+1(n \geq 2)$ dimensions, and $\kappa_{1}, \cdots, \kappa_{n}$ the $n$ principal curvatures at a point $P$ of the hypersurface $V^{n}$. It is known that the $i$ th mean curvature $M_{i}$ of the hypersurface $V^{n}$ at the point $P$ is defined by

$$
C_{n, i} M_{i}=\sum \kappa_{1} \kappa_{2} \cdots \kappa_{i} \quad(i=1, \cdots, n),
$$

where the expression on the right side is the $i$ th elementary symmetric function of $\kappa_{1}, \cdots, \kappa_{n}$, and $C_{n, i}$ denotes the number of combinations of $n$ different things taken $i$ at a time. Let $d A$ be the area element of the hypersurface $V^{n}$ at the point $P$, and $p$ the scalar product of the unit normal vector of the hypersurface $V^{n}$ at the point $P$ and the position vector of the point $P$ with respect to any orthogonal frame in the space $R^{n+1}$.

The purpose of this paper is to prove the following four theorems concerning closed hypersurfaces by first showing that:

a) If $V^{n}$ is an orientable hypersurface, with a closed boundary $V^{n-1}$ of dimension $n-1 \quad(n \geqq 2)$, which is twice differentiably imbedded in an $(n+1)$-dimensional Riemannian space $R^{n+1}$, then the integral $\int_{V^{n}}\left(1+M_{1} p\right) d A$ can be expressed as an integral over the boundary $V^{n-1}$.

b) If in addition $V^{n}$ is of class $C^{3}$ and the space $R^{n+1}$ is of constant Riemannian curvature, then the integral $\int_{V^{\prime 2}}\left(M_{n-1}+M_{n} p\right) d A$ can also be expressed as an integral over $V^{n-1}$.

These results have been obtained in a previous paper [2] by the author for an orientable hypersurface $V^{n}$ twice differentiably imbedded in a Euclidean space $E^{n+1}$ of $n+1(n \geqq 2)$ dimensions.

THEOREM 1. Let $V^{n}$ be a closed orientable hypersurface twice differentiably imbedded in a Riemannian space $R^{n+1}$ of $n+1(n \geq 2)$ dimensions, then

$$
A+\int_{V^{n}} M_{1} p d A=0
$$

Received June 28, 1954 and in revised forms January 3, 1955 and May 10, 1955. 
THEOREM 2. Let $V^{n}$ be a closed orientable hypersurface of class $C^{3}$ imbedded in an $(n+1)$-dimensional $(n \geqq 2)$ Riemannian space $R^{n+1}$ of constant Riemannian curvature $K$, then

$$
\int_{V^{n}} M_{n-1} d A+\int_{V^{n}} M_{n} p d A=0
$$

TheOREM 3. Let $V^{n}$ be a hypersurface satisfying the conditions of Theorem 2. Suppose that the principal curvatures $\kappa_{1}, \cdots, \kappa_{n}$ at each point of the hypersurface $V^{n}$ are positive and that in the space $R^{n+1}$ there exists a point $O$ for which either $p \leqq-1 / M_{1}$ or $p \geqq-1 / M_{1}$ at all points of the hypersurface $V^{n}$. Then every point of the hypersurface $V^{n}$ is umbilic.

THEOREM 4. Let $V^{n}$ be a hypersurface satisfying the conditions of Theorem 2. Suppose that the principal curvatures $\kappa_{1}, \cdots, \kappa_{n}$ at each point of the hypersurface $V^{n}$ are positive and $M_{n-1}$ is constant, and that in the space $R^{n+1}$ there exists a point $O$ for which the function $p$ is of the same sign at all points of the hypersurface $V^{n}$. Then every point of the hypersurface $V^{n}$ is umbilic.

1. Preliminaries. In a Riemannian space $R^{n+1}$ of dimension $n+1$ $(n \geqq 2)$ with a positive definite fundamental form we consider a fixed orthogonal frame $O e_{1} \cdots e_{n+1}$, where $e_{1}, \cdots, e_{n+1}$ form an ordered set of $n+1$ mutually orthogonal contravariant unit vectors at a point $O$ in $R^{n+1}$. With respect to this orthogonal frame let $y^{\alpha}(\alpha=1, \cdots, n+1)$ be ${ }^{1}$ the coordinates of a point in $R^{n+1}$ and $a_{\alpha \beta} d y^{\alpha} d y^{\beta}$ the fundamental form for $R^{n+1}$, where $a_{\alpha \beta}=a_{\beta \alpha}$ and the matrix $\left\|a_{\alpha \beta}\right\|$ is positive definite so that the determinant $\alpha=\left|a_{\alpha \beta}\right|>0$.

Let $A_{i 1}(i=1, \cdots, n)$ be $n$ vectors at a point in the space $R^{n+1}$ whose contravariant components with respect to the frame $O e_{1} \cdots e_{n+1}$ are $A_{i 1}^{\alpha}$ $(\alpha=1, \cdots, n+1)$. First we define the vector product of the $n$ vectors $A_{i 1}(i=1, \cdots, n)$ to be a vector in $R^{n+1}$, denoted by $A_{11} \times \cdots \times A_{n^{\prime}}$, whose contravariant components are given by

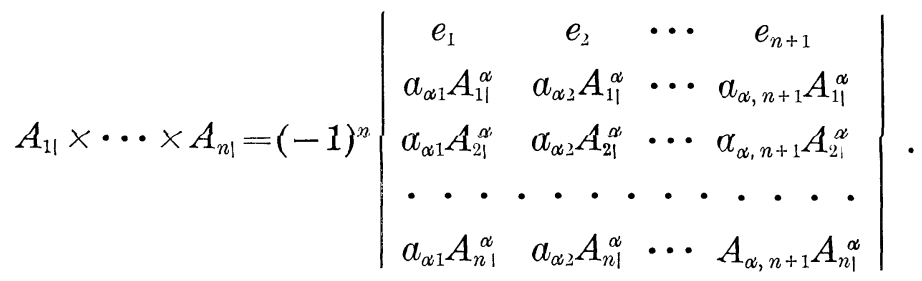

From the definition of the scalar product of any two vectors $A_{i 1}$ and $A_{j l}$,

1 Throughout this paper Greek indices take the values 1 to $n+1$, and Latin indices the values 1 to $n$ unless stated otherwise. We use the convention that repeated indices imply summation. 
namely, $A_{i \mid} \cdot A_{j \mid}=a_{\alpha \beta} A_{i \mid}^{\alpha} A_{j ;}^{\beta}$, it follows immediately that $A_{1 \mid} \times \cdots \times A_{n \mid}$ is orthogonal to $A_{i 1}(i=1, \cdots, n)$.

Now we consider a hypersurface $V^{n}$ twice differentiably imbedded in the space $R^{n+1}$. With respect to the orthogonal frame $O e_{1} \cdots e_{n+1}$ the hypersurface $V^{n}$ can be given by the parametric equations

$$
y^{\alpha}=f^{\alpha}\left(x^{1}, \cdots, x^{n}\right) \quad(\alpha=1, \cdots, n+1),
$$

or the vector equation

$$
Y=F\left(x^{1}, \cdots, x^{n}\right),
$$

where $y^{\alpha}$ and $f^{\alpha}$ are respectively the contravariant components of the two vectors $Y$ and $F$, the parameters $x^{1}, \cdots, x^{n}$ take values in a simply connected domain $D$ of the $n$-dimensional real number space, and $f^{\alpha}\left(x^{1}, \cdots, x^{n}\right)$ is of rank $n$ at all points of $D$. Let the first fundamental form of the hypersurface $V^{n}$ at a point $P$ be

$$
d s^{2}=g_{i j} d x^{i} d x^{j}
$$

where the matrix $\left\|g_{i j}\right\|$ is positive definite so that the determinant $g=\left|g_{i j}\right|>0$, and

$$
\begin{aligned}
& g_{i j}=a_{\alpha \beta} y_{, i}^{\alpha} y_{, j}^{\beta}, \\
& y_{, i}^{\alpha}=\partial y^{\alpha} / \partial x^{i} .
\end{aligned}
$$

Let $A_{\beta i}^{\alpha}$ be a mixed tensor of the second order in the $y$ 's, and a covariant vector in the $x$ 's, as indicated by the Greek and Latin indices. Then following Tucker [3], the generalized covariant derivative of $A_{\beta i}^{\alpha}$ with respect to the $x$ 's is defined as

$$
A_{\beta i ; j}^{\alpha}=\frac{\partial A_{\beta i}^{\alpha}}{\partial x^{j}}+\left\{\begin{array}{c}
\alpha \\
\gamma \delta
\end{array}\right\} A_{\beta i}^{\gamma} y_{, j}^{\delta}-\left\{\begin{array}{l}
\gamma \\
\beta \delta \delta
\end{array}\right\} A_{\gamma i}^{\alpha} y_{, j}^{\delta}-\left\{\begin{array}{l}
k \\
i j
\end{array}\right\} A_{\beta k}^{\alpha},
$$

where the Christoffel symbols $\left\{\begin{array}{l}\alpha \\ \beta \gamma\end{array}\right\}$ with Greek indices are formed with respect to the $a_{\alpha \beta}$ and the $y$ 's, and those $\left\{\begin{array}{c}k \\ i j\end{array}\right\}$ with Latin indices with respect to the $g_{i j}$ and the $x$ 's. It should be noted that the definition of generalized covariant differentiation can be applied to any tensor in the $x$ 's and $y$ 's and that the generalized covariant differentiation of sums and products obeys the ordinary rules. If a tensor is one with respect to the $x$ 's only, so that only Latin indices appear, its generalized covariant derivative is the same as its covariant derivative with respect to the $x$ 's. Moreover, in generalized covariant differentiation the fundamental tensors $a_{\alpha \beta}$ and $g_{i j}$ can be treated as constants. Since $y^{\alpha}$ is an invariant for transformation of the $x$ 's, its generalized covariant derivative 
is the same as its covariant derivative with respect to the $x$ 's; so that

$$
y_{; i}^{\alpha}=y_{, i}^{\alpha}=\partial y^{\alpha} / \partial x^{i} \text {. }
$$

By (1.7) the generalized covariant derivative of $y_{; i}^{\alpha}$ is

$$
y_{; i j}^{\alpha}=\frac{\partial^{2} y^{\alpha}}{\partial x^{i} \partial x^{j}}-\left\{\begin{array}{l}
h \\
i j
\end{array}\right\} y_{, h}^{\alpha}+\left\{\begin{array}{l}
\alpha \\
\beta \gamma
\end{array}\right\} y_{, i}^{\beta} y_{, j}^{\gamma},
$$

which is symmetric in the indices $i$ and $j$.

Let $N$ be the unit normal vector at a point $P$ of the hypersurface $V^{n}$, then

$$
\begin{gathered}
a_{\alpha \beta} N^{\alpha} N^{\beta}=1, \\
a_{\alpha \beta} N_{\alpha} y_{, i}^{\beta}=0
\end{gathered}
$$$$
(i=1, \cdots, n) .
$$

We can easily obtain (see, for instance, [4, Chap. VIII]) :

$$
\begin{aligned}
& y_{; i j}^{\alpha}=\Omega_{i j} N^{\alpha}, \\
& \Omega_{i j}=y_{; i j}^{\alpha} a_{\alpha \beta} N^{\beta}, \\
& N_{; i}^{\alpha}=-\Omega_{i j} g^{j k} y_{, k}^{\alpha},
\end{aligned}
$$

where $\Omega_{i j}=\Omega_{j i}$ are the coefficients of the second fundamental form of the hypersurface $V^{n}$ at the point $P$, and $g^{i j}$ denotes the cofactor of $g_{i j}$ in $g$ divided by $g$ so that

$$
g^{i j} g_{j k}=\delta_{k}^{i},
$$

$\delta_{k}^{i}$ being the Kronecker delta. Moreover, we have

$$
\begin{gathered}
R_{l i j k}=\left(\Omega_{l j} \Omega_{i k}-\Omega_{l k} \Omega_{i j}\right)+\bar{R}_{\beta \gamma \delta \varepsilon} y_{, l}^{\beta} y_{, i, j}^{\gamma} y_{, j}^{\delta} y_{, k}^{\mathrm{\varepsilon}}, \\
\Omega_{i j, k}-\Omega_{i k, j}=-\bar{R}_{\beta \gamma \delta \mathrm{\varepsilon}} N^{\beta} y_{, i}^{\gamma} y_{,,}^{\delta} y_{, k}^{\varepsilon},
\end{gathered}
$$

where $R_{l i j k}$ and $\bar{R}_{\beta \gamma \delta \varepsilon}$ are Riemann symbols formed with the tensors $g_{i j}$ and $a_{\alpha \beta}$ respectively. In particular, if the space $R^{n+1}$ is of constant Riemannian curvature $K$, it follows from the definition of Riemannian curvatures of the space $R^{n+1}$ that

$$
\bar{R}_{\beta \gamma \delta \varepsilon}=K\left(a_{\beta \delta} a_{\gamma \varepsilon}-a_{\beta \varepsilon} a_{\gamma \delta}\right),
$$

and therefore (1.16), (1.17) reduce to

$$
\begin{gathered}
R_{l i j k}=\left(\Omega_{l j} \Omega_{i k}-\Omega_{l k} \Omega_{i j}\right)+K\left(g_{l j} g_{i k}-g_{l k} g_{i j}\right), \\
\Omega_{i j, k}-\Omega_{i k, j}=0 .
\end{gathered}
$$

Taking the generalized covariant derivative of each side of (1.14) and 
making use of (1.12), (1.19), (1.20) we thus obtain

$$
N_{; j i}^{\alpha}-N_{; i j}^{\alpha}=N^{\alpha} g^{\imath k}\left(R_{j i k 1}-\bar{R}_{\beta \gamma \delta \varepsilon} y_{, j}^{\beta} y_{, i}^{\gamma} y_{, k}^{\delta} y_{, l}^{\mathrm{g}}\right) .
$$

The $n$ principal curvatures $\kappa_{1}, \cdots, \kappa_{n}$ of the hypersurface $V^{n}$ at the point $P$ are the roots of the determinant equation

$$
\left|\Omega_{i j}-\kappa g_{i j}\right|=0 \text {. }
$$

From (0.1) and (1.22) it follows immediately that

$$
M_{n}=\Omega / g, \quad n M_{1}=\Omega_{i j} g^{i j}, \quad n M_{n-1}=g_{i j} \Omega^{i j} / g,
$$

where $\Omega=\left|\Omega_{i j}\right|$ and $\Omega^{i j}$ is the cofactor of $\Omega_{i j}$ in $\Omega$.

Consider the two matrices

$$
\phi=\left\|\phi_{\gamma}^{i}\right\|, \quad \psi=\left\|\psi_{i}^{\gamma}\right\| ;
$$

where

$$
\phi_{\gamma}^{i}=a_{\beta \gamma} y_{, i}^{\beta}, \quad \phi_{i}^{\gamma}=y_{, i}^{\gamma} \quad(i=1, \cdots, n ; \gamma=1, \cdots, n+1),
$$

the superscript of the element $\phi_{\gamma}^{i}$ or $\phi_{i}^{\gamma}$ indicating the row to which the element belongs and the subscript indicating the column. Solving (1.11) for $N^{\alpha}$, we obtain

$$
N^{\alpha}=(-1)^{n-\alpha+1} c A^{\alpha} \quad(\alpha=1, \cdots, n+1),
$$

where $c$ is a constant and $A^{\alpha}$ the determinant of $n$th order obtained by deleting the $\alpha$ th column from the matrix $\phi$. Substitution of (1.26) in (1.10) gives

$$
c^{2}=\begin{gathered}
1 \\
a A
\end{gathered},
$$

where

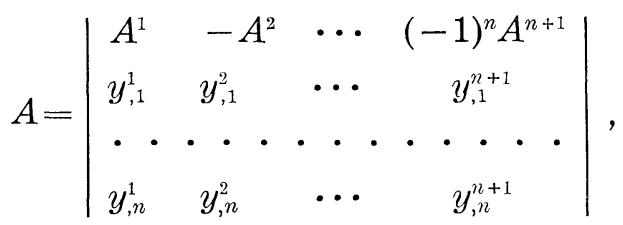

which is equal to the sum of the products of the corresponding determinants of $n$th order of the two matrices (1.24). By an elementary theorem on determinants (see, for instance, [1, p. 102]), from (1.5) it follows immediately that

$$
A=\left|\phi_{\gamma}^{i} \psi_{j}^{\gamma}\right|=g .
$$

Now we choose the direction of the unit normal vector $N$ in such 
a way that the two frames $P Y_{, 1} \cdots Y_{, n} N$ and $O e_{1} \cdots e_{n+1}$ have the same orientation. Then from (1.10), (1.26), (1.27), (1.29) we obtain

$$
\begin{aligned}
& \vee g a N=Y_{, 1} \times \cdots \times Y_{, n}, \\
& \left|Y_{, 1}, \cdots, Y_{, n}, N\right|=V g / a .
\end{aligned}
$$

The area element of the hypersurface $V^{n}$ at the point $P$ is given by

$$
d A=\sqrt{g} d x^{1} \cdots d x^{n} .
$$

Let $A_{i 1}(i=1, \cdots, n)$ be $n$ vectors at a point in the space $R^{n+1}$, whose contravariant components with respect to the frame $O e_{1} \cdots e_{n+1}$ are differentiable functions of $x^{1}, \cdots, x^{n}$, then by (1.1) and the differentiation of determinants

(1.33) $\left(A_{1 \mid} \times \cdots \times A_{n 1}\right)_{; i}=\sum_{j}\left(A_{1 \mid} \times \cdots \times A_{j-1 \mid} \times A_{j \mid ; i} \times A_{j+1 \mid} \times \cdots \times A_{n \mid}\right)$.

2. Proof of the formula (I). First we observe that the vector $Y{ }_{1} \times \cdots \times Y,_{i-1} \times N \times Y,_{i+1} \times \cdots \times Y_{,_{n}}$ is orthogonal to the normal vector $N$ and can therefore be written in the form

$$
Y,_{1} \times \cdots \times Y_{,_{i-1}} \times N \times Y,_{i+1} \times \cdots \times Y_{{ }_{n}}=c^{i j} Y,{ }_{j} \quad(i=1, \cdots, n) .
$$

Taking the scalar products of both sides of (2.1) with the vector $Y_{{ }_{k}}$ and making use of (1.2), (1.5), (1.31), we obtain

$$
c^{i j} g_{j k}=-\sqrt{ } g a \delta_{k}^{i} \quad(i, k=1, \cdots, n) .
$$

Solving (2.2) for $c^{i j}$ for each fixed $i$ and substituting the results in (2.1), we are led to

$$
Y,{ }_{1} \times \cdots \times Y_{{ }_{i-1}} \times N \times Y,_{i+1} \times \cdots \times Y,_{n}=-V g a g^{i j} Y,{ }_{j} \quad(i=1, \cdots, n) .
$$

Making use of the relation $Y_{; i j}=Y_{; j i}$ and (1.14), (1.23), (1.30), (1.33), it is easily seen that

$$
\begin{aligned}
\sum_{i}\left(Y_{{ }_{1}}\right. & \left.\times \cdots \times Y_{,_{i-1}} \times N \times Y_{,_{i+1}} \times \cdots \times Y_{{ }_{n}}\right)_{; i} \\
& =\sum_{i} Y_{{ }_{1}} \times \cdots \times Y_{,_{i-1}} \times N_{; i} \times Y_{,_{i+1}} \times \cdots \times Y,_{n_{n}} \\
& =-n \vee g a M_{1} N .
\end{aligned}
$$

Thus, from (2.3) and (2.4),

$$
n \vee g M_{1} N=\left(\sqrt{ } g g^{i j} Y_{, i}\right)_{; j} .
$$

Taking the scalar products of both sides of (2.5) with the vector $Y$, we obtain in consequence of (1.5) and (1.15) 


$$
n M_{1} p \vee g=\left(\vee g g^{i j} \eta_{i}\right)_{; j}-n \vee g,
$$

where we have put

$$
p=Y \cdot N, \quad \eta_{i}=Y \cdot Y,{ }_{i} \quad(i=1, \cdots, n) .
$$

Now let us consider a hypersurface $V^{n}$, with a closed boundary $V^{n-1}$ of dimension $n-1(n \geqq 2)$, twice differentiably imbedded in a Riemannian space $R^{n+1}$ of dimension $n+1$. Integrating (2.6) with respect to $x^{1}, \cdots, x^{n}$ over this hypersurface $V^{n}$ and applying the general theorem of Stokes to the first term on the right side of (2.6), we obtain

$$
A+\int_{V^{i 2}} M_{1} p d A=\frac{1}{n} \int_{V^{n-1}} \sum_{j}(-1)^{j-1} V g g^{i j} \eta_{i} d x^{1} \cdots d x^{j-1} d x^{j+1} \cdots d x^{n} .
$$

In particular, when the hypersurface $V^{n}$ is closed and orientable, the integral on the right side of (2.8) vanishes and hence we obtain the formula (I).

3. Proof of the formula (II). For the same reason as in the preceding section, the vector $N_{; 1} \times \cdots \times N_{; i-1} \times N \times N_{; i+1} \times \cdots \times N_{; n}$ is orthogonal to the normal vector $N$ and can therefore be written in the form

$$
N_{; 1} \times \cdots \times N_{; i-1} \times N \times N_{; i+1} \times \cdots \times N_{; n}=c^{i j} Y,{ }_{j} \quad(i=1, \cdots, n) .
$$

Taking the scalar products of both sides of (3.1) with the vector $Y,{ }_{k}$ and making use of (1.1), (1.14), (1.31), we obtain

$$
\begin{aligned}
& c^{i j} g_{j k}=(-1)^{n+i} a\left|N, Y,{ }_{1}, \cdots, Y,{ }_{n}\right|
\end{aligned}
$$

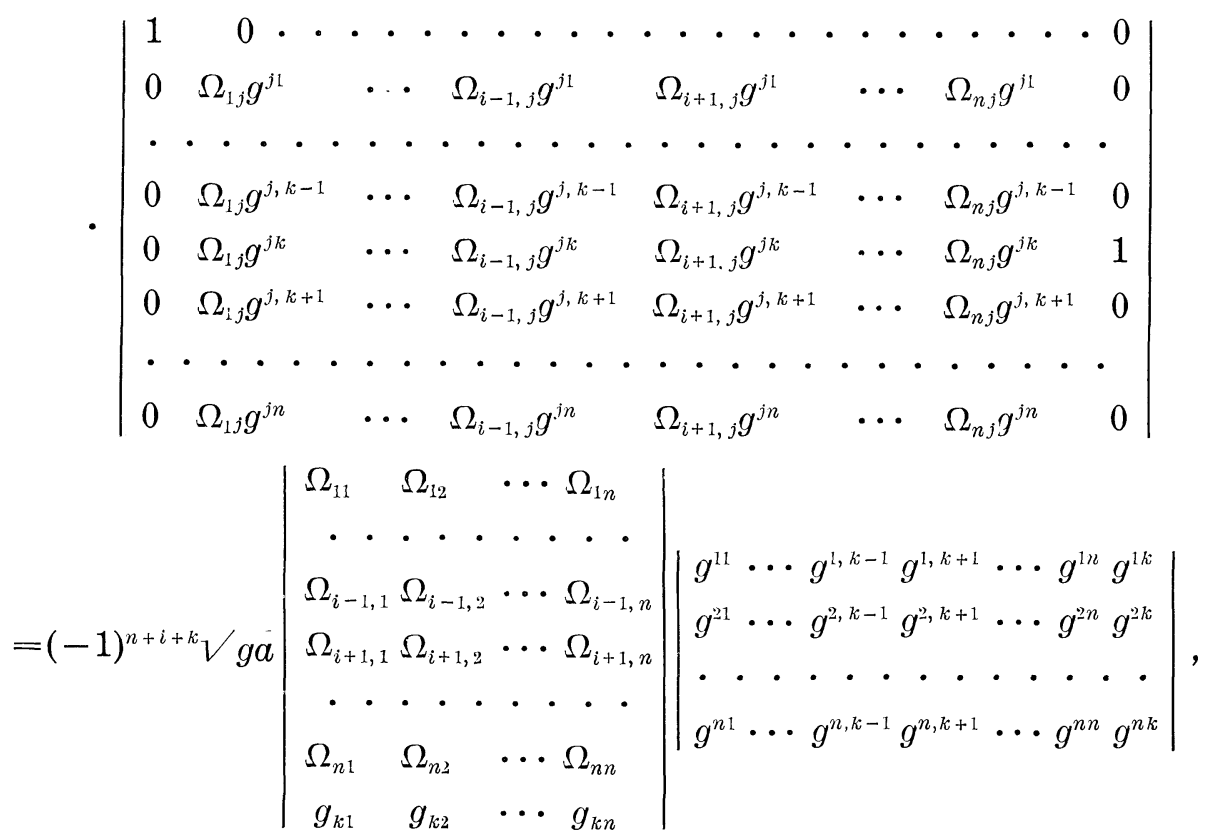


and therefore

$$
c^{i j} g_{j k}=(-1)^{n} \sqrt{a / g} g_{k j} \Omega^{i j} \quad(i, k=1, \cdots, n) .
$$

Solving (3.2) for $c^{i j}$ for each fixed $i$ and substituting the results in (3.1), we find

$$
N_{; 1} \times \cdots \times N_{; i-1} \times N \times N_{; i+1} \times \cdots \times N_{; n}=(-1)^{n} \sqrt{a / g} \Omega^{i j} Y,{ }_{j} .
$$

Making use of (1.1), (1.14), (1.21), (1.23), (1.30), (1.33), it is easily seen that

$$
\begin{aligned}
& \sum_{i}\left(N_{; 1} \times \cdots \times N_{; i-1} \times N \times N_{; i+1} \times \cdots \times N_{; n}\right)_{; i} \\
& =\sum_{i}\left(N_{; 1} \times \cdots \times N_{; i-1} \times N_{; i} \times N_{; i+1} \times \cdots N_{; n}\right)
\end{aligned}
$$

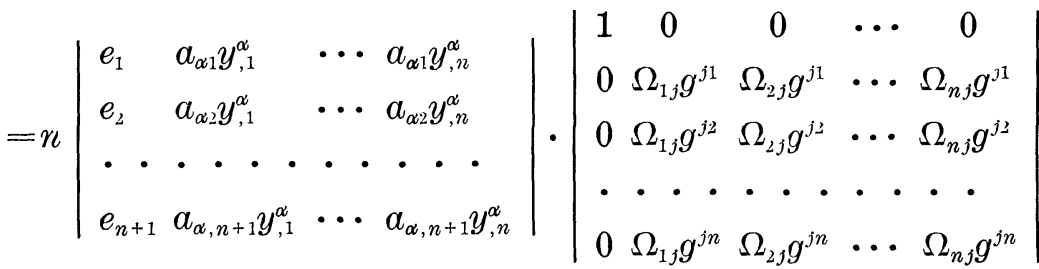

$$
\begin{aligned}
& =(-1)^{n} n \bigvee g a M_{n} N \text {. }
\end{aligned}
$$

Thus, from the above equation and (3.3),

$$
n \vee g M_{n} N=\left(\Omega^{i j} Y,{ }_{j} / V g\right)_{; i} .
$$

Taking the scalar products of both sides of (3.4) with the vector $Y$, we obtain in consequence of (1.23) and (2.7)

$$
n M_{n} p \vee g=\left(\Omega^{i j} \eta_{j} / V g\right)_{; i}-n M_{n-1} \sqrt{ } g .
$$

As in the preceding section, let us consider a hypersurface $V^{n}$, with a closed boundary $V^{n-1}$ of dimension $n-1(n \geq 2)$, differentiably of class $C^{3}$ imbedded in an $(n+1)$-dimensional Riemannian space $R^{n+1}$ of constant Riemannian curvature $K$. Integrating (3.5) with respect to $x^{1}, \cdots, x^{n}$ over this hypersurface $V^{n}$ and applying Stokes' theorem to the first term on the right side of (3.5), we then obtain

$$
\begin{aligned}
\int_{V^{n}} M_{n-1} d A+\int_{V^{n}} M_{n} p d A \\
\quad=\frac{1}{n} \int_{V^{n-1}} \sum_{j}(-1)^{j-1} \frac{\Omega^{i j} \eta_{j}}{\sqrt{g}} d x^{1} \cdots d x^{j-1} d x^{j+1} \cdots d x^{n} .
\end{aligned}
$$

In particular, when the hypersurface $V^{n}$ is closed and orientable, the integral on the right side of (3.6) vanishes and hence the formula (II). 
4. Proofs of Theorems 3 and 4. For $M_{1}>0$, the assumptions $p \leqq-1 / M_{1}$ and $p \geqq-1 / M_{1}$ are respectively equivalent to $1+M_{1} p \leqq 0$ and $1+M_{1} p \geqq 0$. From formula (I) it follows that each of the above two assumptions implies that $p=-1 / M_{1}$. Substituting this in (II) we obtain

$$
\int_{V^{n}} \frac{1}{M_{1}}\left(M_{1} M_{n-1}-M_{n}\right) d A=0,
$$

which holds when and only when $M_{1} M_{n-1}-M_{n}=0$, since

$$
\begin{aligned}
M_{1} M_{n-1}-M_{n} & =\frac{1}{n^{2}}\left(\sum \kappa_{i} \sum \kappa_{i_{1}} \kappa_{i_{2}} \cdots \kappa_{i_{n-2}}-n^{2} \kappa_{1} \kappa_{2} \cdots \kappa_{n}\right) \\
& =\frac{1}{n^{2}} \sum\left[\kappa_{i_{1}} \kappa_{i_{2}} \cdots \kappa_{i_{n-2}}\left(\kappa_{i_{n-}}{ }^{1-} \kappa_{i_{n}}\right)^{2}\right] \geqq 0,
\end{aligned}
$$

where $i_{1}, i_{2}, \cdots, i_{n}$ are distinct and run from 1 to $n$. From $(4.1),(4.2)$ it follows that $\kappa_{1}=\kappa_{2}=\cdots=\kappa_{n}$ at each point of the hypersurface $V^{n}$ and therefore that the quantity defined by

$$
\left(\Omega_{i j} q^{i} q^{j}\right) /\left(g_{i j} q^{i} q^{j}\right)
$$

at each point of the hypersurface $V^{n}$ for an arbitrary direction $q$ in the hypersurface $V^{n}$ with contravariant components $q^{i}$ is independent of the direction $q$. Hence $\Omega_{i j}=c g_{i j}$ for all $i$ and $j$ at each point of the hypersurface $V^{n}$, where $c$ is a scalar invariant, so that every point of the hypersurface $V^{n}$ is umbilic.

If $M_{n-1}$ is constant, multiplying the formula (I) by $M_{n-1}$ and subtracting the formula (II) by the resulting equation we obtain

$$
\int_{V^{n}} p\left(M_{1} M_{n-1}-M_{n}\right) d A=0 \text {. }
$$

From this and the assumption that $p$ is of the same sign at all points of the hypersurface $V^{n}$, Theorem 4 follows by exactly the same argument as above.

\section{REFERENCES}

1. L. P. Eisenthart, Riemannian geometry, Princeton, 1926.

2. C. C. Hsiung, Some integral formulas for closed hypersurfaces, Math. Scand., 2 (1954), 286-294.

3. A. W. Tucker, On generalised covariant differentiation, Ann. of Math., 32 (1931), 451-460.

4. C. E. Weatherburn, An introduction to Riemannian geometry and the tensor calculus, Cambridge, 1938. 



\section{PACIFIC JOURNAL OF MATHEMATICS}

EDITORS

\author{
H. L. Royden \\ Stanford University \\ Stanford, California \\ E. Hewite \\ University of Washington \\ Seattle 5 , Washington
}

\author{
R. P. Dilworth \\ California Institute of Technology \\ Pasadena 4, California
}

\author{
A. Horn* \\ University of California \\ Los Angeles 24, California
}

\section{ASSOCIATE EDITORS}
E. F. BECKENBACH
M. HALL
M. S. KNEBELMAN
J. J. STOKER
C. E. BURGESS
P. R. HALMOS
I. NIVEN
V. GANAPATHY IYER
T. G. OSTROM
G. SZEKERES
H. BUSEMANN
R. D. JAMES
M. M. SCHIFFER
F. WOLF
H. FEDERER

\section{SPONSORS}

\author{
UNIVERSITY OF BRITISH COLUMBIA \\ CALIFORNIA INSTITUTE OF TECHNOLOGY \\ UNIVERSITY OF CALIFORNIA \\ CALIFORNIA RESEARCH CORPORATION \\ MONTANA STATE UNIVERSITY \\ UNIVERSITY OF NEVADA \\ OREGON STATE COLLEGE \\ UNIVERSITY OF OREGON \\ UNIVERSITY OF SOUTHERN CALIFORNIA
}

\author{
STANFORD UNIVERSITY \\ UNIVERSITY OF UTAH \\ WASHINGTON STATE COLLEGE \\ UNIVERSITY OF WASHINGTON \\ AMERICAN MATHEMATICAL SOCIETY \\ HUGHES AIRCRAFT COMPANY
}

Mathematical papers intended for publication in the Pacific Journal of Mathematics should be typewritten (double spaced), and the author should keep a complete copy. Manuscripts may be sent to any of the editors. Manuscripts intended for the outgoing editors should be sent to their successors. All other communications to the editors should be addressed to the managing editor, Alfred Horn at the University of California, Los Angeles 24, California.

50 reprints of each article are furnished free of charge; additional copies may be obtained at cost in multiples of 50 .

The Pacific Journal of Mathematics is published quarterly, in March, June, September, and December. The price per volume (4 numbers) is $\$ 12.00$; single issues, $\$ 3.50$. Back numbers are available. Special price to individual faculty members of supporting institutions and to individual members of the American Mathematical Society: $\$ 4.00$ per volume; single issues, $\$ 1.25$.

Subscriptions, orders for back numbers, and changes of address should be sent to Pacific Journal of Mathematics, c/o University of California Press, Berkeley 4, California.

Printed at Kokusai Bunken Insatsusha (International Academic Printing Co., Ltd.), No. 10, 1-chome, Fujimi-cho, Chiyoda-ku, Tokyo, Japan.

* During the absence of E. G. Straus.

PUBLISHED BY PACIFIC JOURNAL OF MATHEMATICS, A NON-PROFIT CORPORATION COPYRIGHT 1956 BY PACIFIC JOURNAL OF MATHEMATICS 


\section{Pacific Journal of Mathematics}

\section{Vol. 6, No. $2 \quad$ December, 1956}

Louis Auslander, Remark on the use of forms in variational calculations .......................................... 209

Hubert Spence Butts, Jr. and Henry B. Mann, Corresponding residue systems in algebraic number fields ........................ 211

L. Carlitz and John Herbert Hodges, Distribution of matrices in a finite field............................................

Paul Civin and Bertram Yood, Invariant functionals ............... 231

David James Dickinson, Henry Pollak and G. H. Wannier, On a class of polynomials orthogonal over a denumerable set .................

Bernard Friedman and Luna Mishoe, Eigenfunction expansions associated with a non-self-adjoint differential equation ....................

Luna Mishoe and G. C. Ford, On the uniform convergence of a certain eigenfunction series .............................. 271

John W. Green, Mean values of harmonic functions on homothetic curves...........................................

Charles John August Halberg, Jr. and Angus E. Taylor, On the spectra of linked operators .....................................

Chuan Chih Hsiung, Some integral formulas for closed hypersurfaces in Riemannian space ................................... 291

Norman D. Lane, Differentiable points of arcs in conformal n-space 301

Louis F. McAuley, A relation between perfect separability, completeness, and normality in semi-metric spaces

G. Power and D. L. Scott-Hutton, The slow shearing motion of a liquid past a semi-infinite plane .............................

A. C. Schaeffer, Entire functions

Edward Silverman, An intrinsic inequality for Lebesgue area...

Choy-Tak Taam, Asymptotic relations between systems of differential equations.

Ti Yen, Quotient algebra of a finite $A W^{*}$-algebra ... 